\title{
Simulation of Flow Suspended Load in Weirs by Using
}

\section{Flow3D Model}

\author{
Mehdi Taghavi ${ }^{\mathrm{a}^{*}}$, Hesam Ghodousi ${ }^{\mathrm{b}}$ \\ ${ }^{a}$ Master of Science Student, Department of Civil Engineering, Islamic Azad University, Kish Branch, Kish Island, Iran \\ ${ }^{b}$ Assistant Professor, Department of Water Engineering, University of Zanjan, Zanjan, Iran.
}

Received 25 October 2015; Accepted 28 November 2015

\begin{abstract}
Sharp-crested weir and side-channel weirs can be considered among the control and flow-measuring structures which are greatly applicable in channels, dam spillways and other hydraulic structures. Discharge coefficient in these two types of weirs is affected by the structural geometry and the hydraulics of the flow. Suspended load is one of the main factors which may affect the hydraulics of the flow and consequently modify the flow discharge coefficient. Using different geometrical and hydraulic conditions, the discharge coefficient relation in sharp-crested and side channel weirs for the case of suspended load can be extracted. Flow3D numerical model is capable of precisely simulation the flow containing suspended and bed load in the main channel. The discharge coefficient equation is deduced and presented based on the Froude number, by numerical iterations. The numerical results reveal that the discharge coefficient in the two cases of clear water and the flow containing suspended load are totally different. At the same hydraulic load, the discharge coefficient in the case of existing suspended load is greater than the case of clear water; In addition, the discharge coefficient is reduced by increasing the Froude number in all cases with different load concentrations.
\end{abstract}

Keywords: Flow3D, Discharge Coefficient, Side-Channel Weir, Sharp-Crested Weir.

\section{Introduction}

The weir is defined as an obstacle which is put in front of the flow and makes the water to rise at the back side of the weir. Besides being used as precise discharge measuring devices in irrigation channels and laboratory flumes, the sharp crested weirs are applicable in the upstream flow height and volume increase. Since the hydraulic theory relevant to this weir is considered as the main base for designing the other types of weirs, the sharp-crested weirs are considered to be of great importance.

The flow in sharp-crested weirs is two dimensional and the discharge eqanduation is obtained based on the following assumptions:

- The streamlines on the crest are horizontal

- The Energy loss on the weir is ignored

- The pressure on the weir crest is equal to the atmospheric pressure.

According to the above mentioned assumptions, the energy in the cross section 0 and 1 are considered to be equal (Figure 1); and so a relation is obtained between the discharge rate and the static head over the crest.

\footnotetext{
*Corresponding author: mehditaghaavi@gmail.com
} 


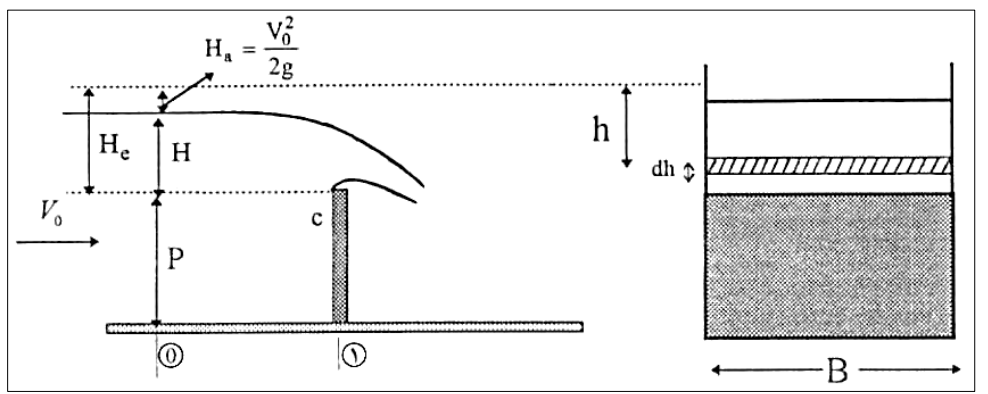

Figure 1. A view of rectangular sharp-crested weir without load contraction

A rectangular element with the width " $\mathrm{B}$ " and the thickness "dh" which has a distance " $h$ " from the energy line is considered; thus, the velocity inside the element would be $v=\sqrt{2 g h}$, and the discharge is $d q=v d q$, so the total discharge in unit width is:

$q=\int_{H_{a}}^{H_{e}} v \cdot d h=\int_{H_{a}}^{H_{e}} \sqrt{2 g h} \cdot d h=\frac{2}{3} \sqrt{2 g}\left(H_{e}^{\frac{3}{2}}-H_{a}^{\frac{3}{2}}\right)=\frac{2}{3} \sqrt{2 g}\left[\left(H+\frac{v_{0}^{2}}{2 g}\right)^{\frac{3}{2}}-\left(\frac{v_{0}^{2}}{2 g}\right)^{\frac{3}{2}}\right]$

Many researchers believe that $\left(\frac{v^{2}}{2 g}\right)$ is small and can be ignored; thus, omitting this term we will obtain:

$Q=\frac{2}{3} \sqrt{2 g} \cdot B \cdot H^{\frac{2}{3}}$

According to the considered assumptions, the actual discharge value is different from the obtained discharge. So, in order to modify the above equation (Eq.2), the empirical coefficient " $C_{d}$ " called the discharge coefficient is used. By using $C_{d}$, equation 1 may be rewritten as follows:

$Q=\frac{2}{3} \sqrt{2 g} \cdot C_{d} \cdot B \cdot H^{\frac{3}{2}}$

Most of the research efforts on the flow over the spillways are relevant to the discharge coefficient determination. Recently, several efforts have been failed to modify the discharge relations, such that the discharge coefficient is not effective in the relations. It should be noted that the non-dimensional coefficient presented in most of the studies have been obtained, considering the conditions at which no suspended load exist.

Side channel weirs, is one of the oldest hydraulic structures, used as a deviating structure in rivers and channels. This type of weir is installed beside the main channel and as the water reaches the crest, the excess water is deviated automatically from the main channel. This structure is greatly applicable in urban drainage and wastewater systems, water supply and irrigation, flow control, flood deviation, and excess discharge of rivers and channels.

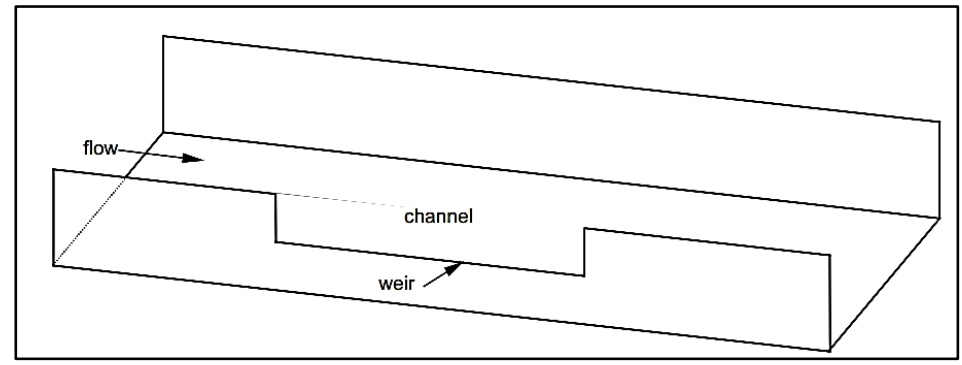

Figure 2. A view of a side-channel weir

Based on the investigations, the suspended load causes a shift in the uniform flow profile compared to the case of clear water. According to Prandtl's Mixing Length theory, the velocity distribution of turbulent flow over smooth and rough bed is described as follows [1]:

$\begin{aligned} \frac{U}{U_{*}} & =\frac{2.303}{K} \log \left(\frac{U_{*} \cdot y}{v}\right)+5.5 \\ \frac{U}{U_{*}} & =\frac{2.303}{K} \log \left(\frac{y}{K_{s}}\right)+8.5\end{aligned}$ 
Where " $U$ " is the flow velocity in distance " $y$ " from the bed, " $U_{*}$ " the flow shear velocity, " $v$ " the kinematic viscosity, " $K$ " the Karman constant (which is 0.4 for clear water) and " $K_{s}$ " is Nikuradse roughness coefficient [2].

Experimental studies of Vanoni (1946), Ismail (1952), and Barton and Lin (1955) revealed the fact that, Karman coefficient is not equal to 0.4 in most of the cases [3]. Einstain \& Chien (1954) showed that the suspended load may reduce the Karman coefficient [4]. It was presented by Vanoni and Nomicos (1960) that the Karman coefficient is decreased when suspended load is carried by the flow [5].

Investigating the effect of velocity profile characteristics on the suspended load concentration change, Coleman $(1981,1986)$, showed that the Karman coefficient is independent of suspended load and mentioned that the traditional theory which relates the reduction of $K$ by the increase in suspended load, is due to the incorrect data analysis method $[6,7]$.

By the study of the suspended load effect on the velocity profile in a flume with the two conditions of smooth and rough beds, Kereselidze and Kutavaia (1995) realized that the velocity in the smoothed bed flume is decreased close to the bed and increased near the free surface and therefore, the suspended load carrying flow average velocity in the smoothed bed flume may be smaller than, equal to or greater than the clear water flow velocity. While in the roughed bed flume, the suspended load carrying flow velocity is increased both close to the bed and near the free surface.

Gohari, Asadi et al. (2005) investigated the effect of the suspended load on discharge coefficient of the side-channel and sharp-crested weirs. The results show that in both structures, the discharge coefficient is affected by the flow suspended load, e.g. decreased by the suspended load increase [9].

As it was observed earlier, the diversity of side-channel and sharp-crested weirs and also different sediment types has caused the experimental study to be time consuming and costly. The researchers have used the Flow3D numerical model in many hydraulic fields such as water quality sedimentation, investigating the sedimentation and erosion in the channel bed and the bridge's piers, the study of the energy dissipation and cavitation calculation of the water free surface profile, velocity and pressure distribution, and combination of different hydraulic structures, e.g. compound spillways. In this study, the flow over sharp-crested and side-channel weirs has been investigated and $R N G$ and $k-\epsilon$ turbulence models have been used for the turbulence field.

\section{Research Methodology}

Flow3D numerical model is applicable for three dimensional flows and is particularly capable of free surface flow analysis. In this software package, Finite Volume method is used to discretize the governing equations over a regular rectangular mesh system. Five turbulence models can be implemented during the simulations which are Prandtl's Mixing Length, One equation models, Two equation models, $k-\epsilon$ and $R N G$, and Large eddy simulation. This software is a powerful modeling tool which provides valuable engineering views of the physical phenomenon. In addition to the special capability in prediction of the free surface flows, Flow3D is ideal software in engineering design and optimized production process. One of the most significant capabilities of this numerical model is the sediment transport modules, which can be used to simulate both cases of suspended and bed loads.

In this software, two numerical techniques are applied. The first is the Volume of Fluid "VOF" presented by Hirt and Nichols (1981) which is used to simulate the free surface [10]. The "VOF" method contains three parts: surface location display, mesh system, and free surface boundary conditions. The second technique, the Fractional AreaVolume Obstacle Representation "FAVOR" is used to simulate the rigid area and volumes as geometric boundaries. One of the advantages of using Flow3D is the user friendly feature of the software (compared to the other software packages) Implementing capability of the solid boundaries and mesh in a more easy way, containing useful help messages in manual to provide more efficient simulation, and automatic selection of the best time interval leading to the solution convergence compared to the other similar software packages. Superiority of this package to the other software packs is due to the efficient technical supports which the developer company provides.

In order to construct the three-dimensional geometry of the model consisting of the main channel and side-channel weirs in Flow3D, a geometry simulating software package like AutoCAD, CATIA, SolidWorks, etc. may be applied. For this purpose, SolidWorks 2011 software package has been used in this study. The flow filed simulation was accomplished in two steps for sharp-crested and side-channel weir. First of all, the sharp-crested weir under a laboratory flume conditions which can be sloped and is $0.3 \mathrm{~m}$ wide, $0.45 \mathrm{~m}$ high, $10 \mathrm{~m}$ long, and has the maximum discharge of $12.5 \mathrm{lit} / \mathrm{s}$, and slope of 0.001 was used. A rectangular sharp-crested weir has the same width as the noncontracted flume. Figure 3 presents characteristics of the laboratory flume of side-channel and sharp-crested weirs. 


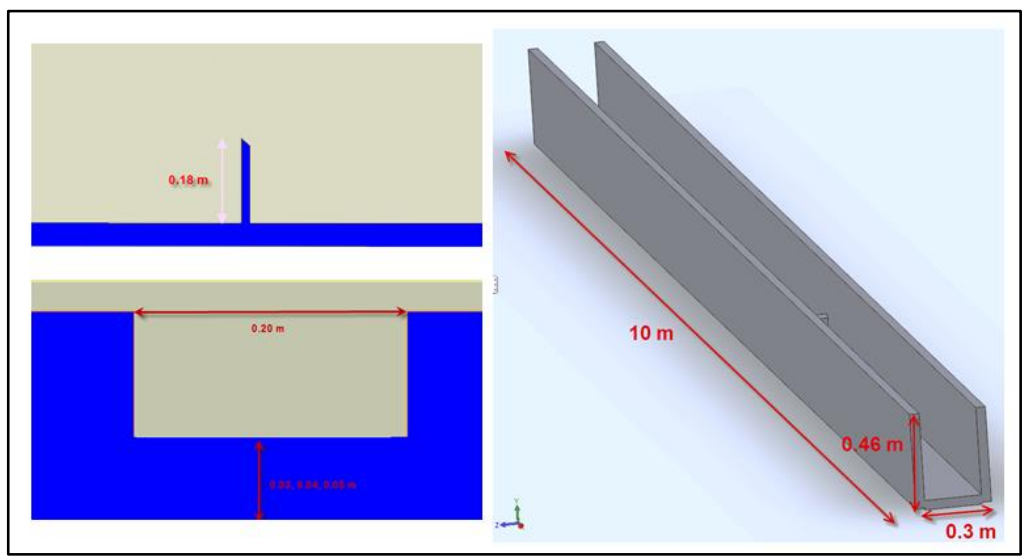

Figure 3. Characteristics of the laboratory flume of the side-channel and sharp-crested weirs

\section{Simulation of the flow field in Flow3D}

In this study, the flow was simulated over non-eroding bed by using existing experimental data, and under the nonviscos incompressible air flow conditions with the density and shear stress coefficient of $1.2 \mathrm{~kg} / \mathrm{m}^{3}$ and 0.073 respectively. All of the simulation and collaboration process have been carried considering the similar laboratory conditions. The first step in a numerical simulation is to choose the calibrated model.

One of the most important advantages of the Flow3D numerical model compared to the other models of flow field simulation is the capability of estimation and determination of the best mesh system for the solution field based on the geometry of the model. In order to determine the mesh dimensions of the computational field in the presented model, the computational cells dimensions toward the longitude, lateral and height $(X \times Y \times Z)$ were considered to be $4.7 \mathrm{~cm}$. Calibrating dimensions of the cells by using the module of FAVOR specified that the dimensions of the selected cells are not appropriate for the main channel and weir geometry. In other words, the whole of the existing geometry is not covered by $4.7 \mathrm{~cm}$ cells and parts of the channel and sharp-crested weir are not included in the model. Making the cells smaller up to $3.5 \mathrm{~cm}$ was shown not to be enough to cover the weir. After investigation and calibration of the dimensions, it was observed that some of the cells introduced to the model are not appropriate. Similar to the previous steps, the cells dimensions are obtained to be optimized to $2.7 \mathrm{~cm}$. Overall, about 10,000 meshes were considered for the above mentioned model. Different conditions for computational cell dimensions have been provided by Figure 4 .

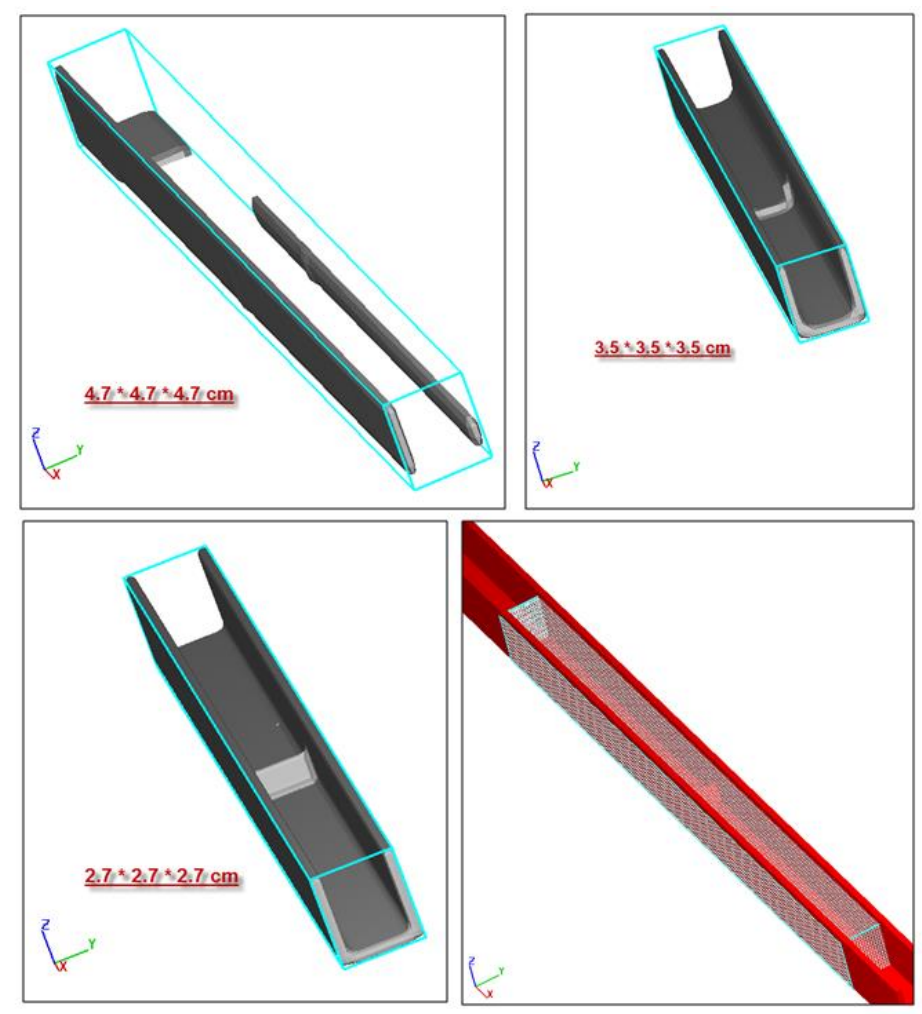

Figure 4. Different mesh systems and calibration mesh selection for the model geometry 
The calibration of the numerical model regarding the boundary conditions has been described so far. In order to extract the precise and correct numerical or experimental data, it is necessary to make the model reach the stable conditions. In the existing numerical study, the suitable running time was considered to be equal to 50 computational steps. Figure 5 presents how the flow passes over the bed in different time intervals. The flow will get stable after 15 computational steps. Figure 5 shows different stages of time steps.
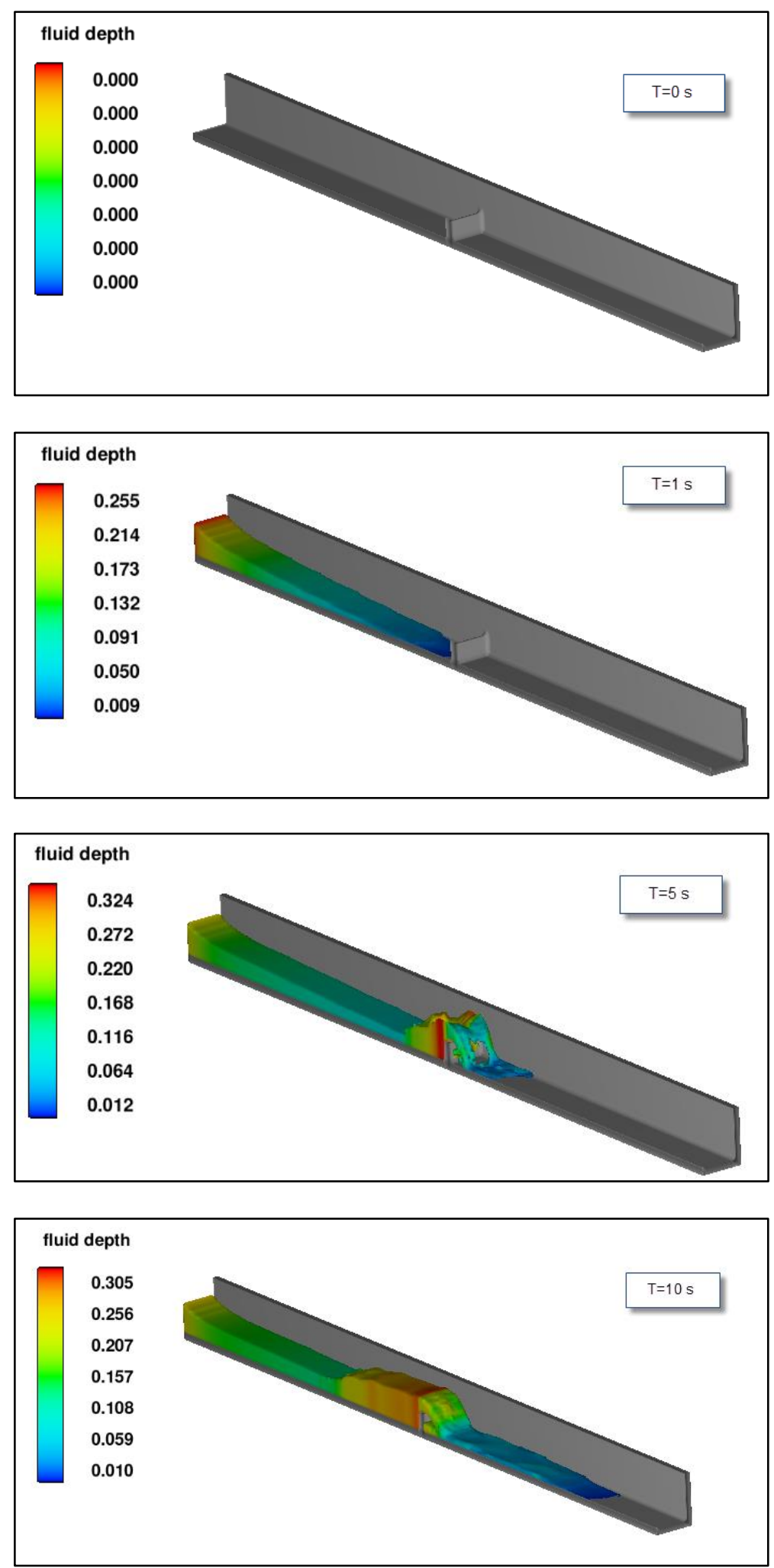

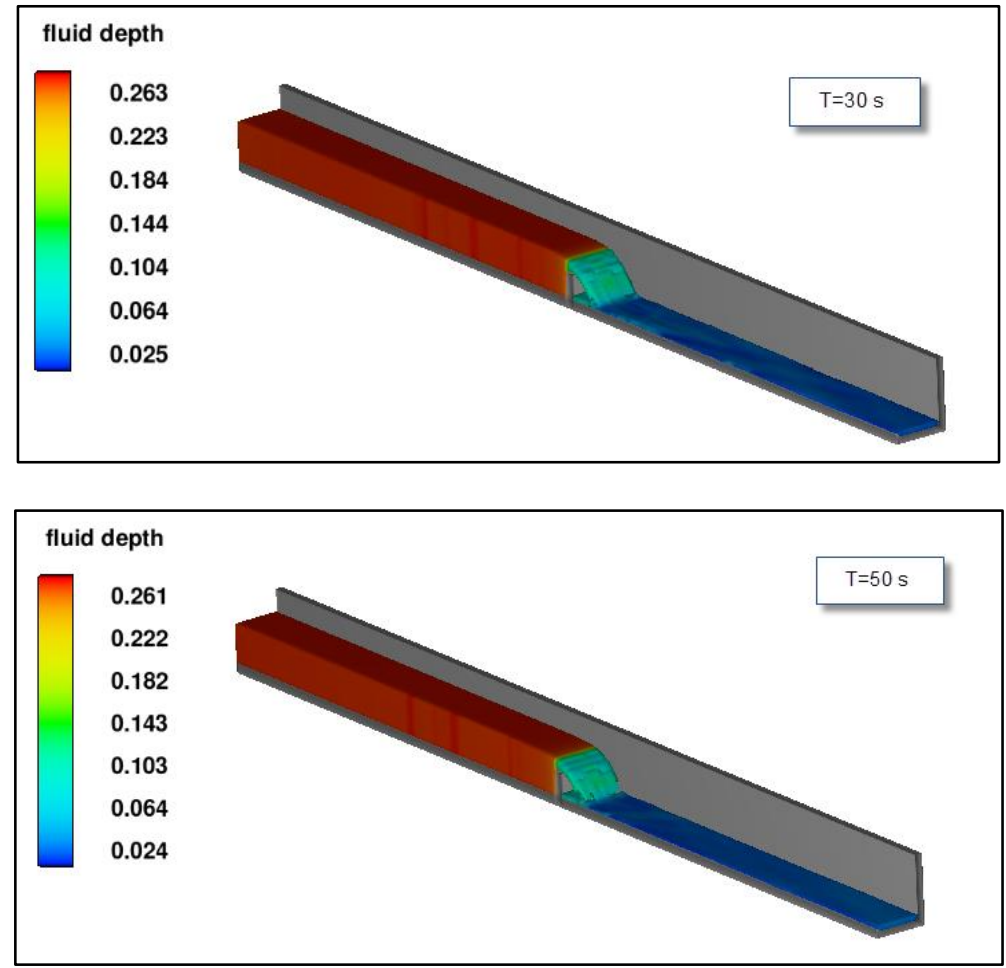

Figure 5. Different computational time steps of the flow stability inside the channel during the 3D simulation

Figure 6 and 7 provide the variations of the output and input discharges. As presented by the figures, after 50 seconds, the total output and input discharges coming from the input and output boundaries have been approximately constant along the channel and the flow has reached to the stable conditions.

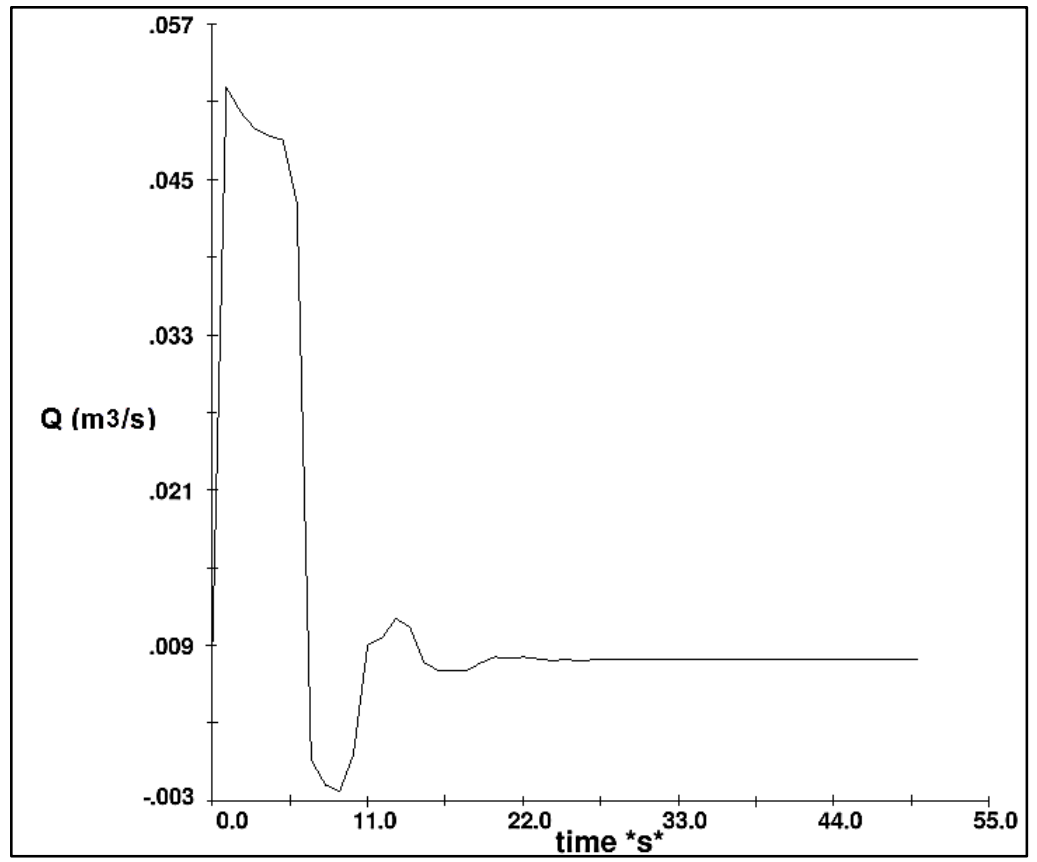

Figure 6. Discharge value from the input boundary and the stable flow inside the channel 


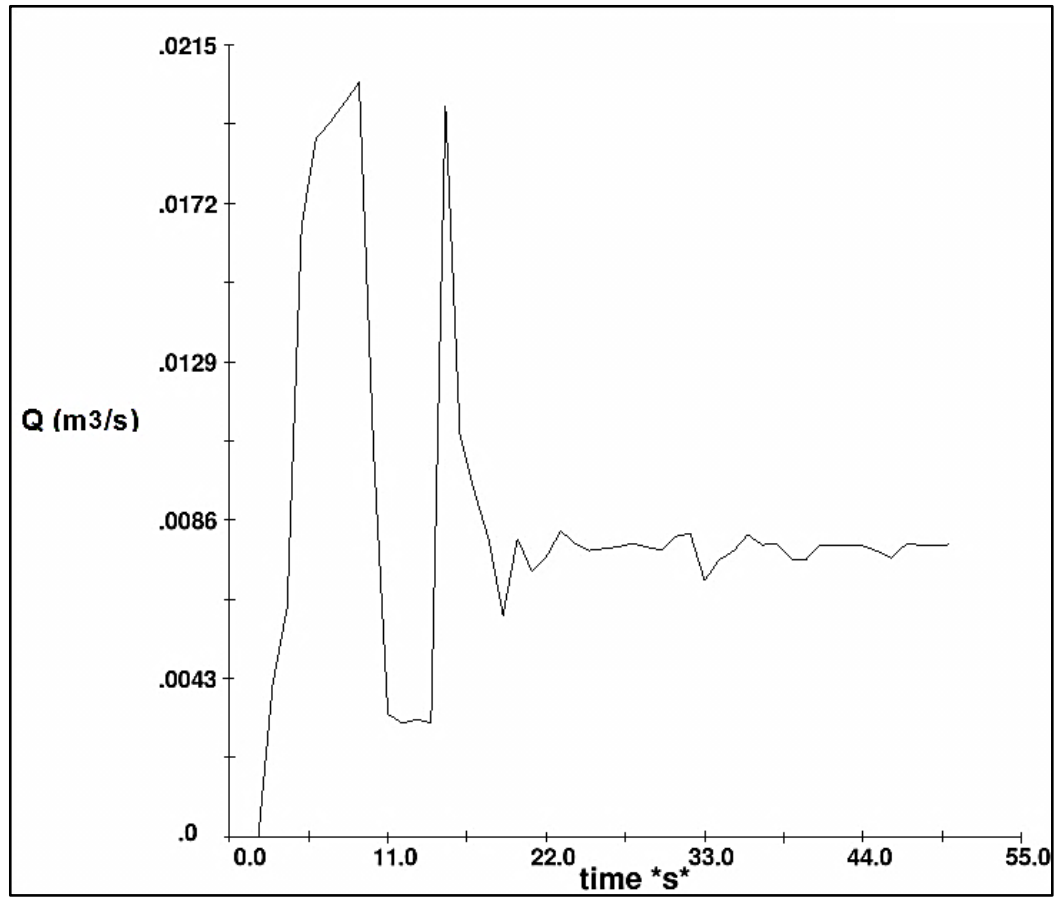

Figure 7. Discharge value from the output boundary and the stable flow inside the channel

\section{Results of the suspended load flow, numerical modelling}

As a first step, in order to investigate the sharp-crested weir discharge coefficient, the sediment material is considered to be the same as the sediment material in the laboratory. For the sharp-crested weir, saw dust with $G_{s}=1.3$ and $D_{50}=0.192 \mathrm{~mm}$ is used. According to the experimental reports saw dust is the most appropriate material which can simulate the suspended load conditions. The grading carves for the saw dust and Silica powder as suspended load materials have been provided by figures 8 and 9 .

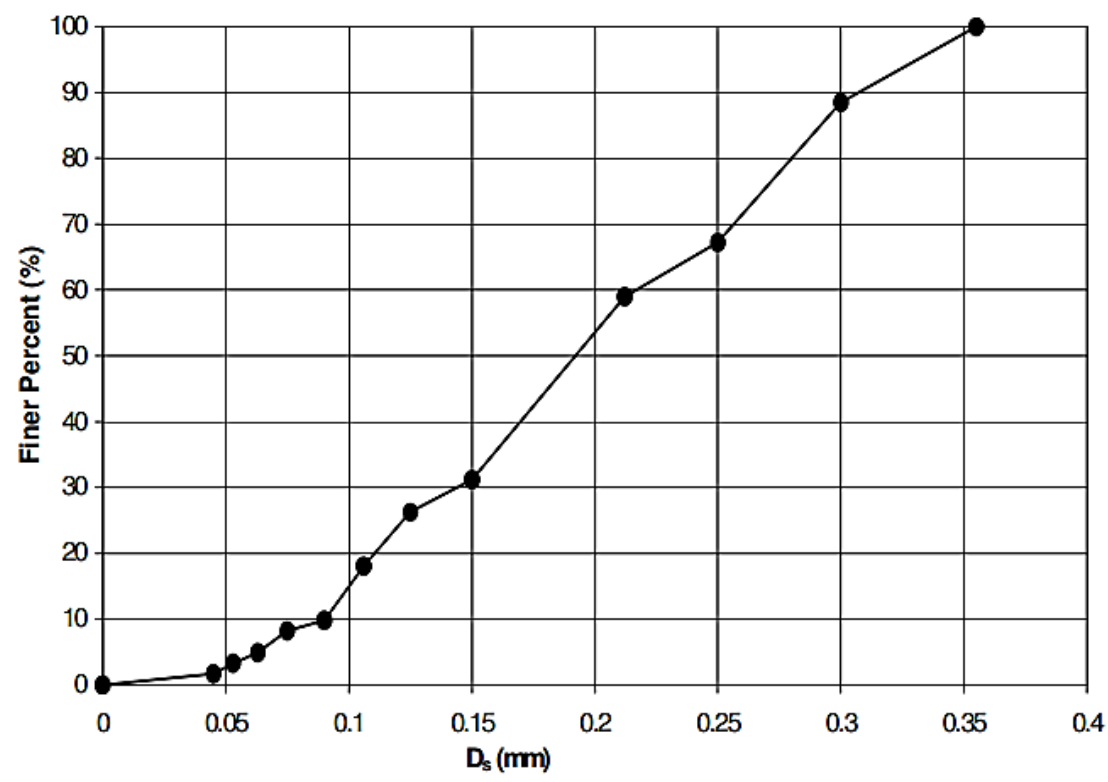

Figure 8. Grading curve of saw dust as the suspended load in sharp-crested weirs 


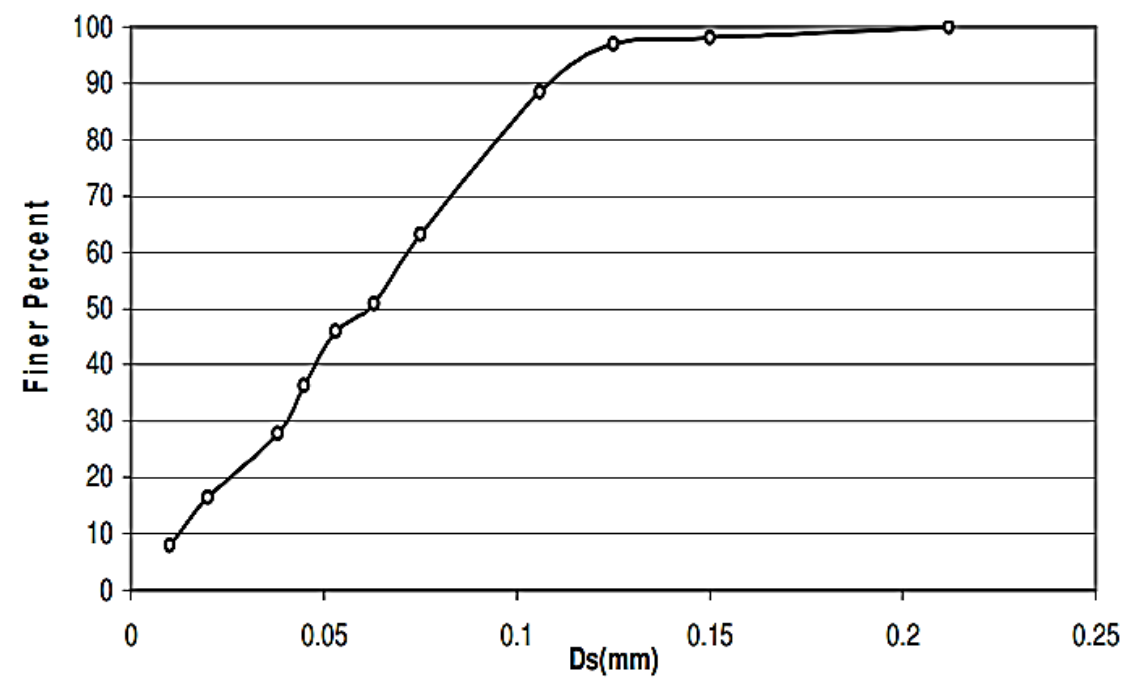

Figure 9. Grading curve of Silica powder as the suspended load in side-channel weirs

Using numerical models, one can extract the flow hydraulic parameters such as the velocity, depth, and pressure toward different directions. Figure 10 presents the pressure in side-channel and sharp-crested weirs.

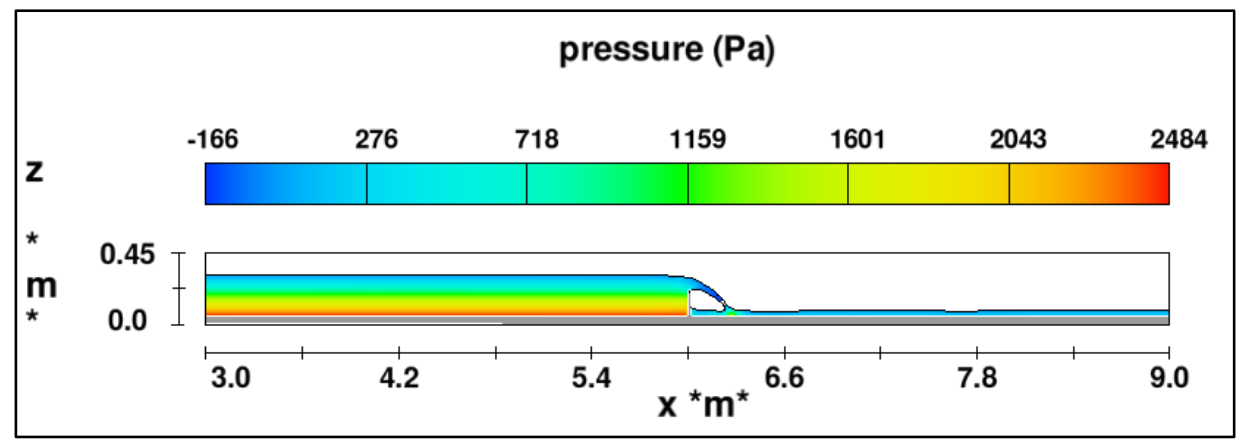

Figure 10. The current pressure along the channel length in sharp-crested weir

As it is observed thorough figure 10, the maximum pressure values which occurs just at the back side of the weir, may be related to the deeper flow existing at the backside of the weir. Figure 11 shows the flow depths at the backside and in front of the sharp-crested weirs.

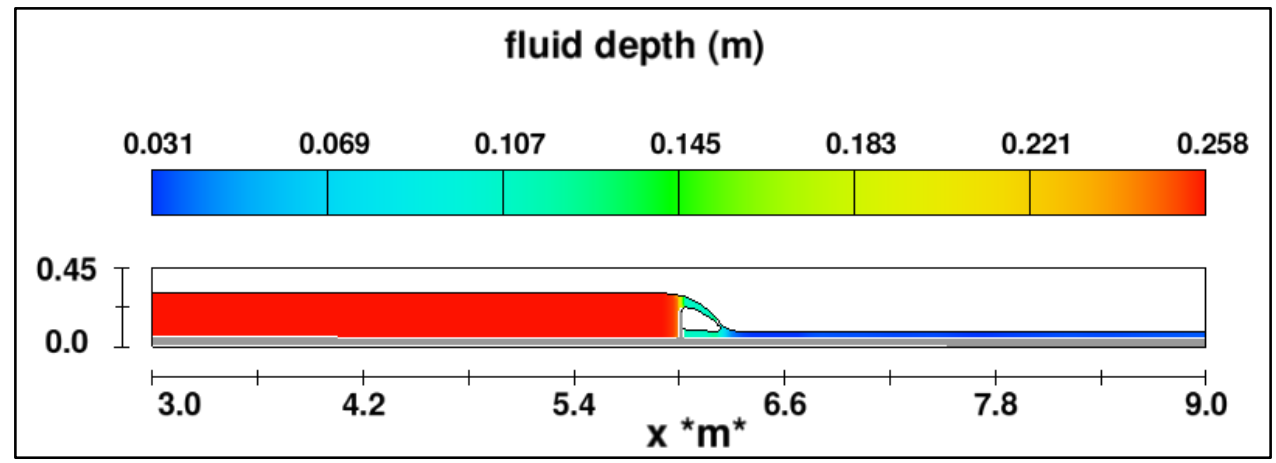

Figure 11. The flow depth values along the channel in the longitudinal direction in sharp-crested weir

Figure 12 provides the flow velocity in the longitudinal direction. The negative values of the velocity are due to the circular flow at the sharp- crested weir downstream. Figure 13 presents the velocity vectors at the downstream of the weir which indicates the existence of rotational flow. Figure 14 and 15 presents the suspended load distribution and the sediment accumulation at the backside of the sharp-crested weir, and at the bottom of the channel. 


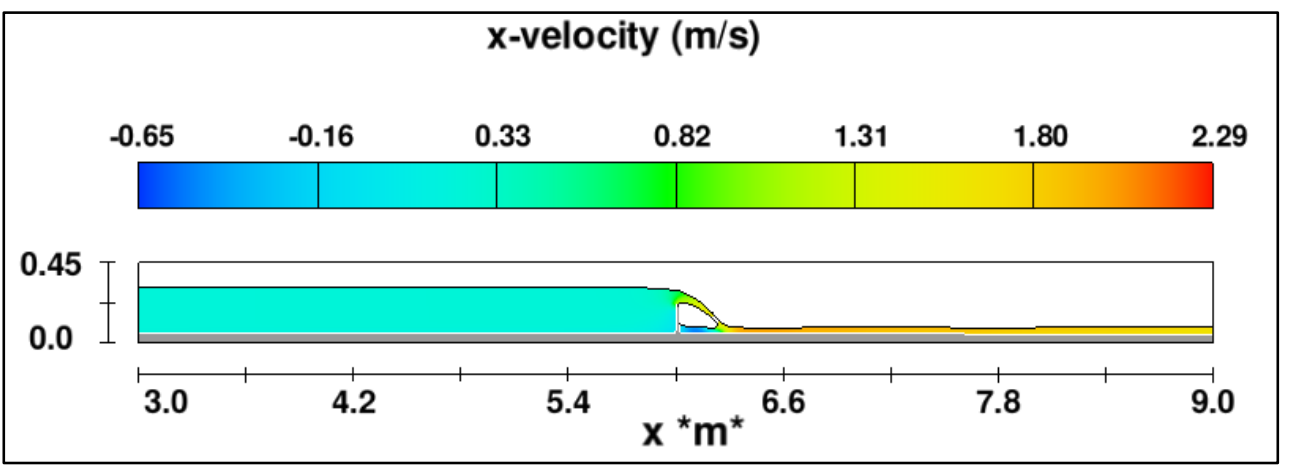

Figure 12. The values of flow velocity in the longitudinal direction of the flow in sharp-crested weirs

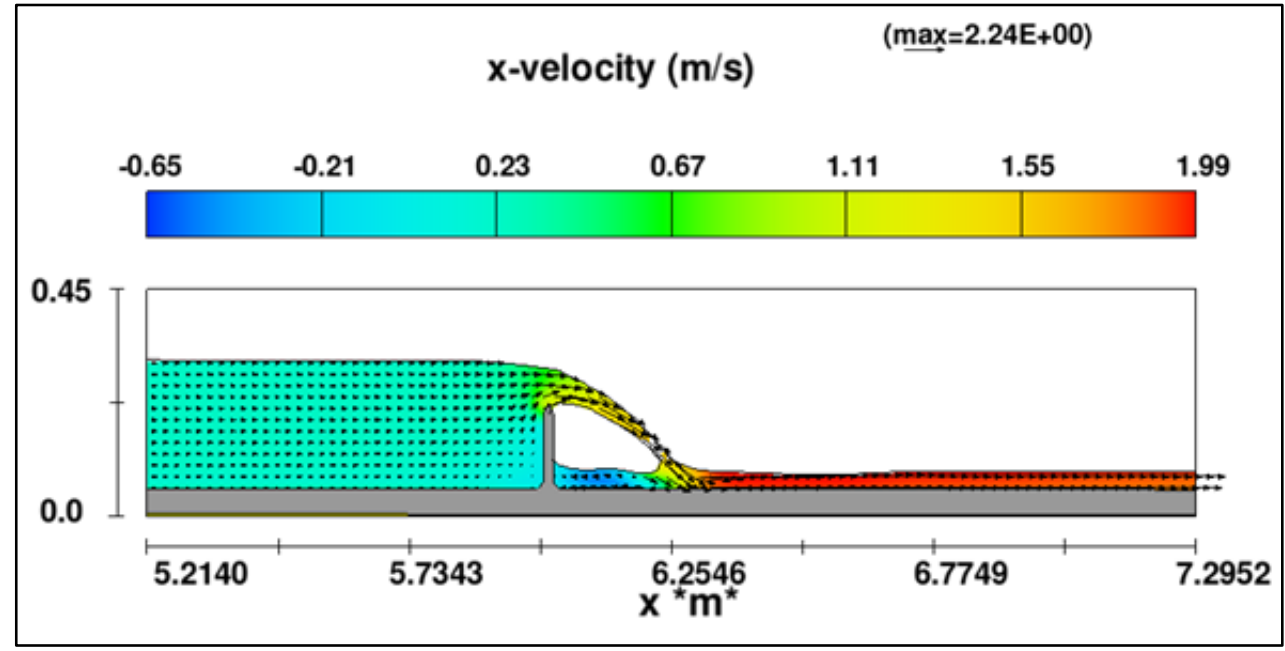

Figure 13. The values of flow velocity together with the velocity vectors in the longitudinal direction of the flow in sharpcrested weirs

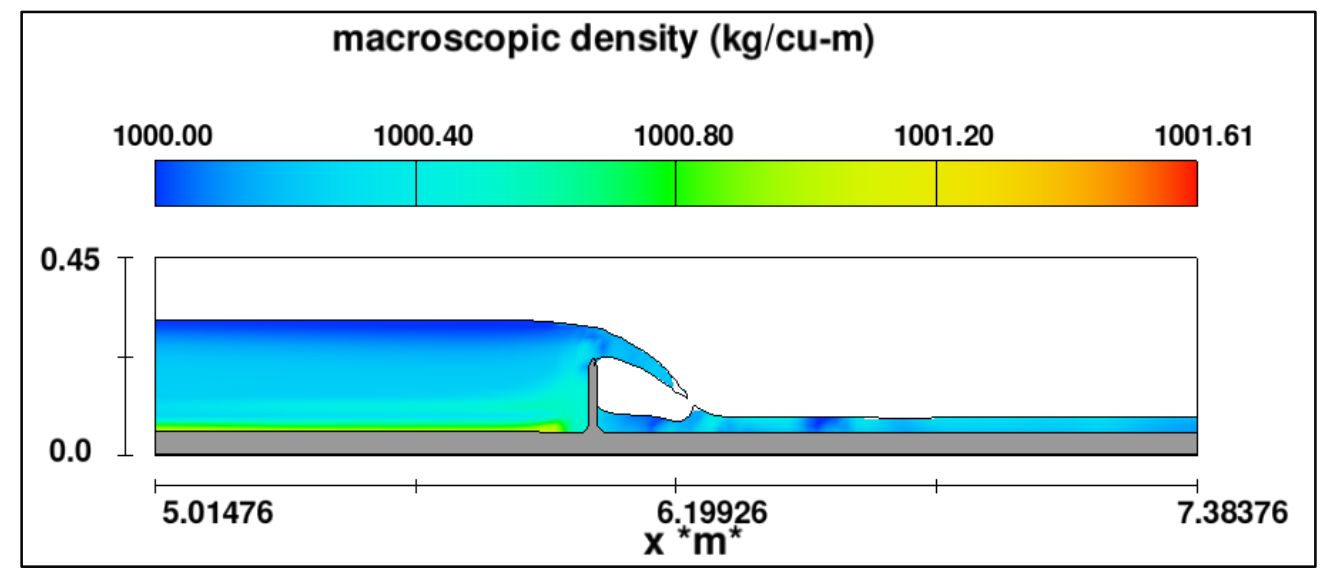

Figure 14. Density variations at the backside of sharp-crested weir and values of the suspended load 


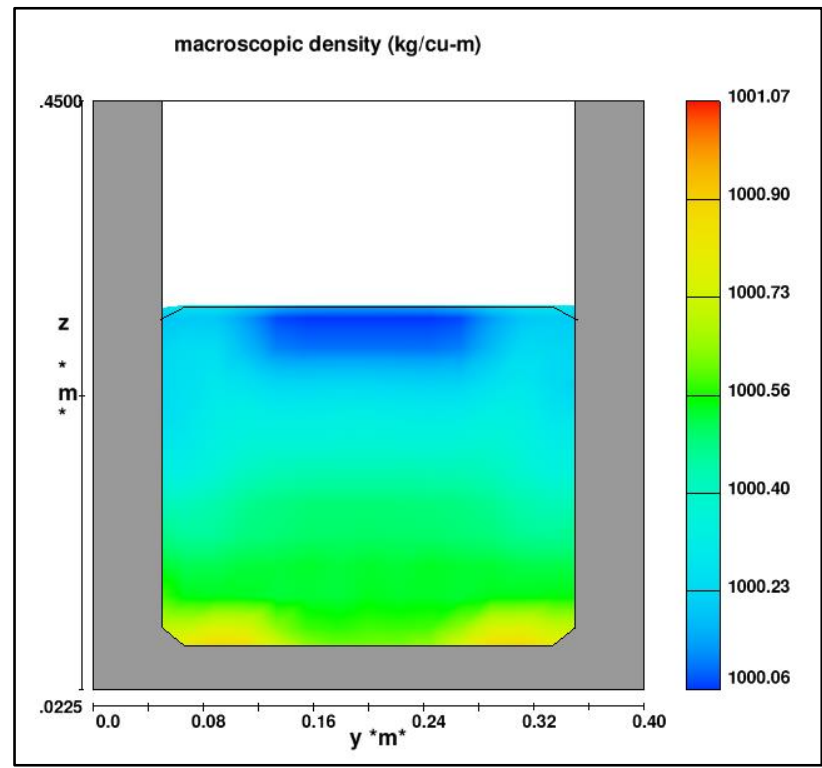

Figure 15. Density variation at the backside section of the sharp-crested weir

Based on the experimental reports, the flow suspended load has been accumulated at the backside of the sharpcrested weir. In the present numerical model, just as the experimental model, the suspended load has been accumulated at the backside of the sharp-crested weir which is recognised by the density variation. It should be noted that the maximum flow density for the saw dust is $1001.07 \mathrm{~kg} / \mathrm{m}^{3}$ extracted from the numerical model.

The 3D simulation of the sharp-crested weirs is presented in the following. Different hydraulic parameters may be extracted from the numerical model. Figure 16 presents the flow depth values for the side channel weir.

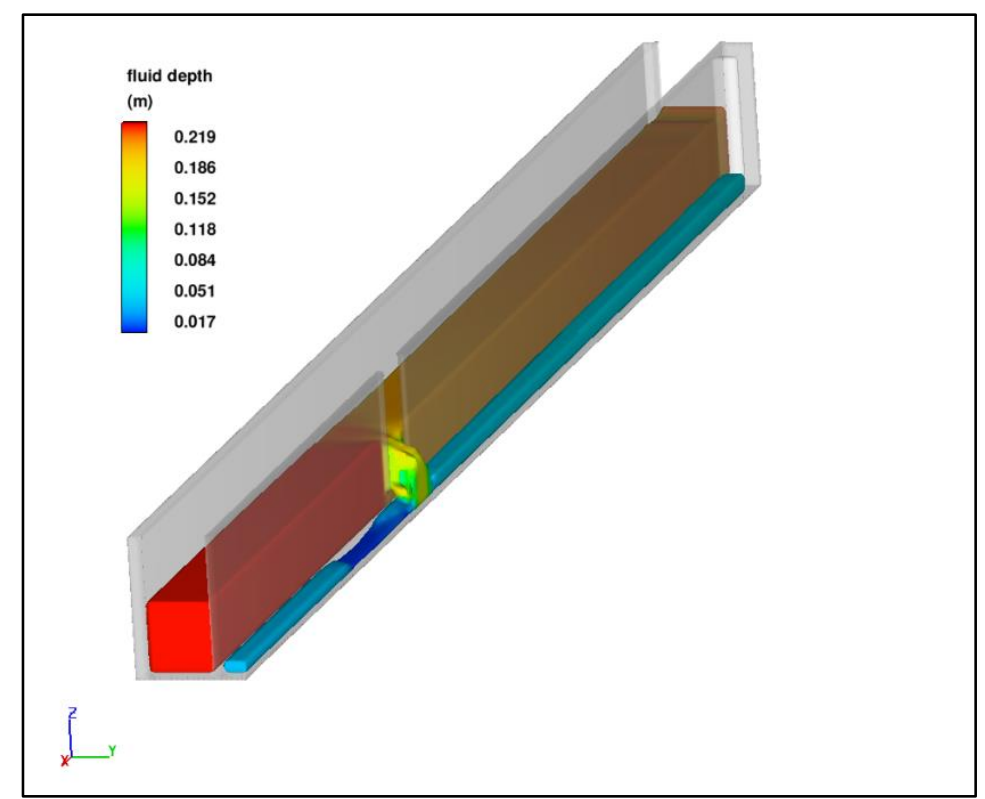

Figure 16. The simulated flow depth values of side channel weirs

As mentioned in the laboratory experiment section, the main channel is divided into two channels of 20 and $10 \mathrm{~cm}$ wide where the side channel weir was put inside. Figures 17 and 18 provide the velocity values extracted from the numerical model. 


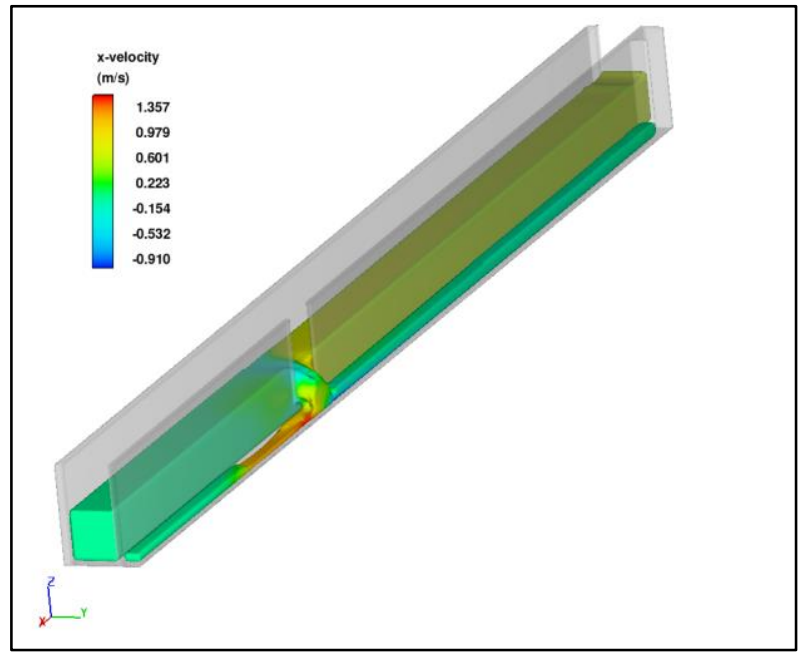

Figure 17. The flow simulated velocity values of side channel weirs

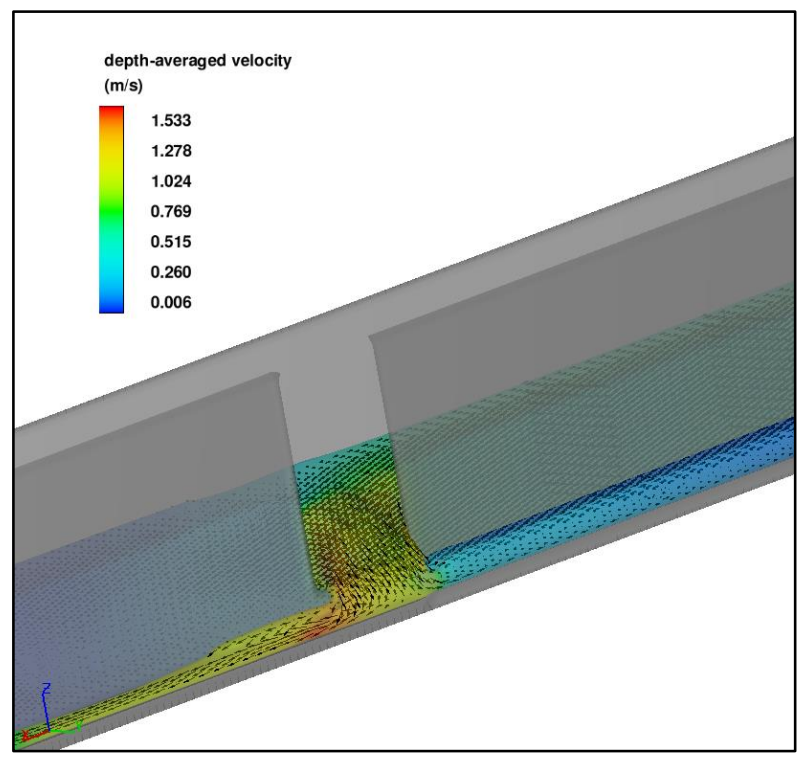

Figure 18. The flow simulated velocity vectors of side channel weirs

Figure 19 presents the velocity vectors based on the velocity contours in the mean depth.

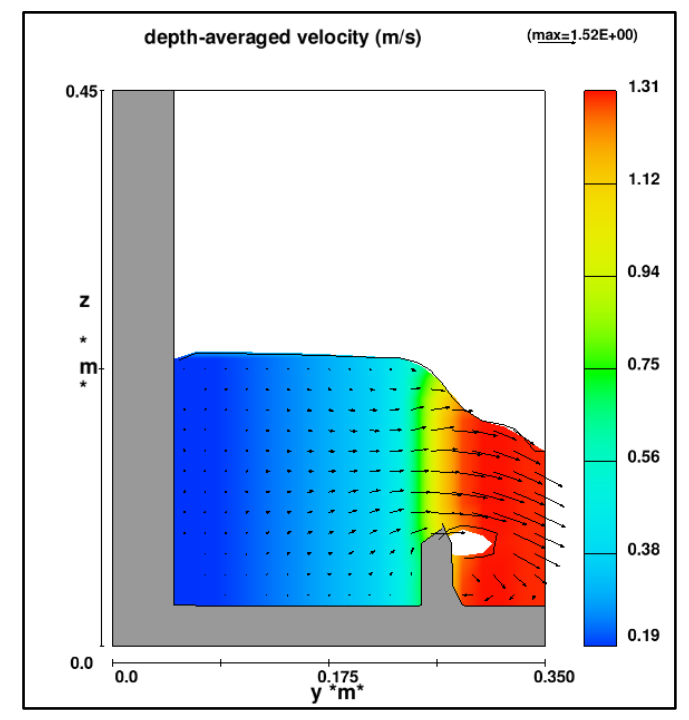

Figure 19. The values of flow velocity in the mean depth with the velocity vectors 


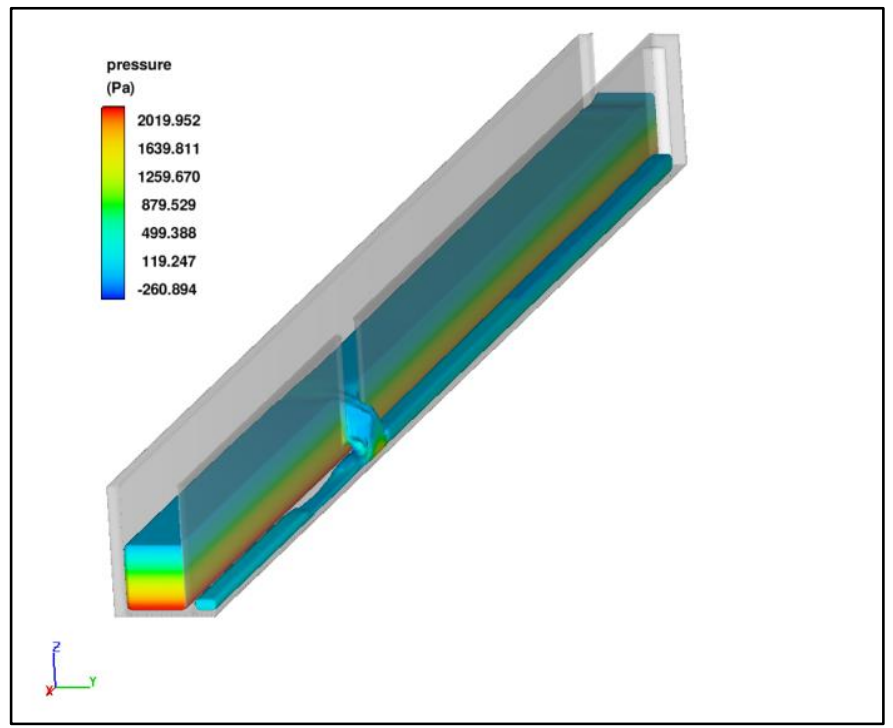

Figure 20. The 3d simulated pressure values of side channel weirs

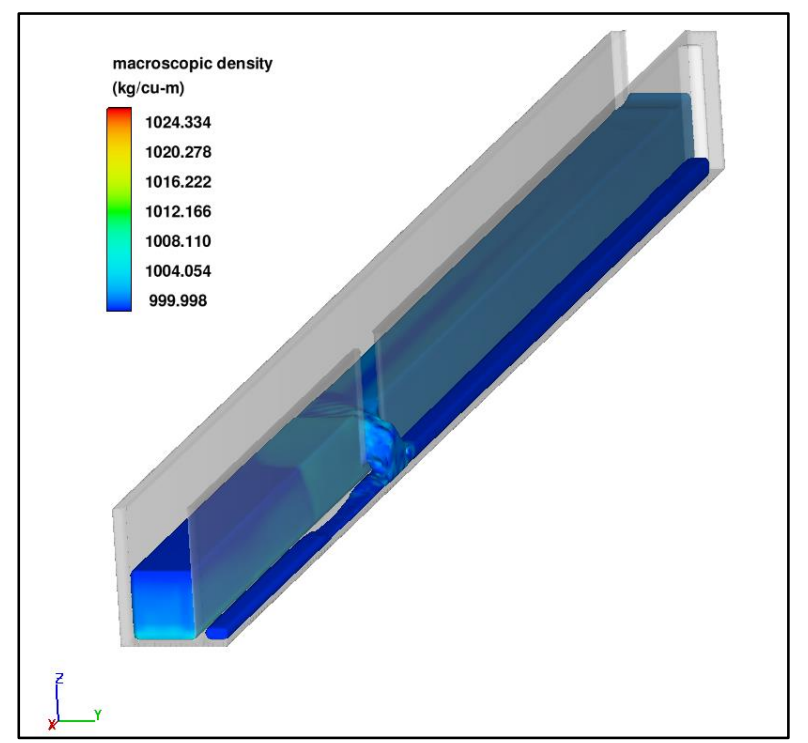

Figure 21. The 3D simulated density values of side channel weirs

Based on the results of the numerical model, the density value is $1024.3 \mathrm{~kg} / \mathrm{m}^{3}$ when using Silica powders, which is greater than the density when sawdust is applied because in the latter, $G_{S}$ has been considered to be $G_{S}=2.65$.

\section{Conclusion}

The presents study has been carried to investigate the suspended load effects on the hydraulic conditions of rectangular sharp-crested and side channel weirs. Implementing numerical simulations, based on the experimental model conditions, the result of numerical model was concluded and compared for the two cases of clear water and suspended load. Compassion of the weir discharge coefficient in the two afore-mentioned cases, reveal that the coefficients are not the same for the two cases. According to the simulations, the discharge coefficient is greater in the case of suspended load rather than in the case of clear water. The maximum flow concentration for the sharp-crested weirs containing sawdust as suspended load and the side-channel weirs containing Silica powders is 1001.7 and $1024.3 \mathrm{~kg} / \mathrm{m}^{3}$ respectively. The simulation results show the appropriate distribution of the suspended load across the depth, while such distribution is not obtained in the experimental tests which are due to the difficulty of the suspended load injection into the whole flow. This can easily accomplished in the numerical model by definition of the concentration value to the model through the input boundaries which is the remarkable advantage of the numerical models in simulating such flows. 


\section{References}

[1]. Yang, Chih Ted. Sediment transport: theory and practice. New York: McGraw-hill, 1996.

[2]. Nikuradse, J. "Strömungsgesetze in rauhen Rohren VDI-Forschungsheft, Bd. 361." (1933).

[3]. Garde, Ramchandra J., and KG Ranga Raju. Mechanics of sediment transportation and alluvial stream problems. Taylor \& Francis, 2000.

[4]. Einstein, H. A., and N. Chien. "Similarity of distorted river models with unstable bed." In Proceedings of the ASCE, vol. 80, no. 566 , p. 21. 1954.

[5]. Vanoni, Vito A., and George N. Nomicos. "Resistance properties of sediment-laden streams." Transactions of the American Society of Civil Engineers 125, no. 1 (1960): 1140-1167.

[6]. Coleman, Neil L. "Velocity profiles with suspended sediment." Journal of Hydraulic Research 19, no. 3 (1981): 211-229.

[7]. Coleman, Neil L. "Effects of Suspended Sediment on the Open - Channel Velocity Distribution." Water Resources Research 22, no. 10 (1986): 1377-1384.

[8]. Kereselidze, N. B., and V. I. Kutavaia. "Experimental research on kinematics of flows with high suspended solid concentration." Journal of Hydraulic Research 33, no. 1 (1995): 65-75.

[9]. Gohari Asadi, S.; Ayoubzade, A.; Samani, M. V., and Aberi Foroutan, Sh. (2005). Comparing discharge coefficients of normal and lateral overflows with flow suspended load, the 5th hydraulic conference of Iran, Kerman, Bahonar University

[10]. Hirt, Cyril W., and Billy D. Nichols. "Volume of fluid (VOF) method for the dynamics of free boundaries." Journal of computational physics 39, no. 1 (1981): 201-225. 